\title{
A scoping review research on the dynamics managing of Coronavirus disease (COVID-19)
}

\author{
Harith Yas, Azman Hashim International Business School, Universiti Teknologi Malaysia, Johor Bahru, \\ Malaysia, Harith.albayati@yahoo.com,ORCHID: 0000-0001-6511-6248 \\ Ahmed Alkaabi, Electrical \& computer school, RMIT University, Australia, alkaabiahmed87@gmail.com \\ Hamdan M. Al Mansoori, Business School, Aberystwyth University, United Kingdom, hma2@aber.ac.uk \\ Mubarak Masoud, Technology Management and Business, University Tun Hussein Onn Malaysia, \\ uaexm@hotmail.com
}

Ahmad Alessa, Respiratory Therapy Administration, King Abdullah Medical City, Mecca, Saudi Arabia, alessarrt@gmail.com

\begin{abstract}
The coronavirus pandemic also commonly known COVID-19 has affected various parts of the world rendering a negative impact on people's lives and properly as well as the global economy. Several strategies have been devised by scientists and other innovators to address the issue of COVID-19 based on its different dynamics. A scoping review research about the dynamics of COVID-19 was conducted with the aid of the Arksey and O'Malley framework. The systematic searches were mostly conducted in ProQuest, Web of Science (WoS), Google Scholar, and Medline whereby key search word such as COVID19 and coronavirus. The scoping review managed to identify 1,067 documents that were carefully scrutinized based on the provisions of the inclusion and exclusion criteria. The eligibility criteria helped in the elimination of 873 documents leaving a sample of 194 documents. Scoping reviews are considered as new methods of reviewing evidence but are increasingly being adopted as the best ways assessing different research problems or concepts. Due to the high variability in the conduct of scoping reviews, there is need for proper methodological standardization which will help to improve the utility and general strength of the evidence.
\end{abstract}

Keywords: Covid-19, Systematic scoping review, coronavirus, SARS-CoV-2, Epidemiology.

Received: 10.12.2020 Accepted: 19.01.2021 $\quad$ Published: 04.02.2021

\section{INTRODUCTION}

As of June 10, 2020, the severe acute respiratory syndrome coronavirus 2 (SARS-CoV-2) or (Covid-19) pandemic has affected almost 7,343,124 coronavirus cases, deaths 414,127 and recovered 3,620,298 people in 168 countries on five continents. According to WHO (2020), SARS-COV-2 can be acquired through various means but most particularly through inhalation of aerosols originating from an infected person. This therefore indicates that the disease can be spared from person to person or to a group of individuals through respiratory droplets released from the mouth as the person coughs, vomits or sneezes. This justifies the reason to why one of the most recommended prevention mechanisms, is wearing a facemask to avoid inhalation of the respiratory droplets of infected persons (Zimmermann \& Nkenke, 2020). According to Zhong (2020), indicates that COVID-19 has greatly affected different countries across the world whereby various people have died, lost their jobs, and economies have also been greatly affected, among other effects. One of the major negative effects that came along with the new form of coronavirus is the sudden outbreak of pneumonia, which is commonly known as the Coronavirus disease (COVID-19) (McKibbin, \& Fernando, 2020). According to Kar et al. (2020), over 31,000 cases and 636 deaths associated with COVID-19 had been reported in total from different parts of China by 6th February 2020, about. The Chinese government alerted the World Health Organization (WHO) about the deadly disease that had spread in different parts of the country and had led to the death of a significant number of people (WHO, 2020).

Sanders et al. (2020) indicates that by 29th may 2020, over 6.1 million cases of coronavirus disease had been reported in over 213 countries around the world most especially in Italy, South Africa, United States, and the United Kingdom, among many other countries across the world. With the emergence of the deadly coronavirus disease in the different parts of China most especially Wuhan city, the Chinese authorities had to take several actions to prevent further spreading of the virus. Among the most effective strategies that were implemented was the lockdown which was first imposed on Wuhan and later implemented in other cities or towns of China (Chiu et al., 2020). The coronavirus disease attracted much attention from the 
World Health Organization (WHO) and on 30th January 2020, WHO declare the disease as a global emergency hence it required maximum attention from different stakeholders or health experts across the world (Bhuiyan et al., 2020). After being declared as a Public Health Emergency of International Concern (PHEIC) by WHO, the coronavirus disease was ;later declared a global pandemic on 11th March 2020 thereby attracting ,more serious interventions or preventative strategies across several countries (Queensland Health Department, 2020). More guidelines and strategies to base on in controlling the coronavirus disease have been issued by different organizations such as the WHO, the United States (US) Centers for Disease Control and Prevention (CDC), different researchers from China, and the European Centre for Disease Prevention and Control, among other health-based organizations (Tai et al., 2020).

Several journals, scholarly articles, editorials, or reviews, have been published about the new coronavirus disease (COVID-19) most especially concerning the way through which the disease is spread, the impact of COVID-19, and strategies to counter attack the disease, among others (Bartoszko et al., 2020). However, the studies about COVD-19 are still limited hence it's a challenging task for most researchers to establish the rightful or satisfying evidence about the nature of the new coronavirus diseases (Khudhair, Alsaud, Alsharm, Alkaabi, \& AlAdeedi, 2020). However, a comprehensive review of the different studies conducted in the past about COVID-19 and other related pandemics could help to increase knowledge about this disease. Most of the first studies about COVID-19 were published in Chinese by different Chinese researchers but due to the rapid spread of the virus, other additional studies, journals, or articles have been published in other countries across the world and can be accessed from different websites or databases (Hall, Scott, \& Gössling, 2020).

\section{A SCOPING REVIEW AND RESEARCH}

Most researchers have defined scoping reviews as the most appropriate tool to use in mapping existing evidence about a particular research problem (Sharma et al., 2020). Scoping review helps to establish different characteristics of selected literature, helps to organize key concepts of the literature and enabling the research to establish the relationship between the key concepts as well as enabling analysis of the literature in order to fill the existing gaps (Arksey and O'Malley, 2005). The scoping review tool has continuously undergone improvements is among the most preferred methodologies for analyzing secondary data or literature review and it employs different techniques associated with systematic reviews and Meta-Analyses (PRSIMA), which helps in making well drafted scoping reviews (Saadat, Rawtani, \& Hussain, 2020). Due to the complexity and urgency of the COVID-19 pandemic as well as the increasing need to understand the different dynamics of the new coronavirus disease, a scoping review was considered the most appropriate tool to use in such a scenario. This paper therefore presents a scoping review of corona virus whereby great attention is put on reviewing different studies that have been conducted in the past about the dynamics of the new coronavirus disease. This scoping review research was also conducted to help identify research gaps related to this new viral disease and propose recommendations for future research on COVID-19.

\section{METHODS}

This particular review research about the dynamics of COVID-19 adopted the scoping review method that was developed by Arksey and O'Malley (2005) to help in reviewing different past studies about a particular research element. The scoping review method involves five steps of establishing the rightful documents to include in the review process. The five steps include; identification of the research question, identifying the most relevant articles or studies, sorting and charting the extracted data, summarizing the obtained results, and reporting the review findings. The process undertaken in gathering evidence for this particular scoping review research is presented in figure 1. 


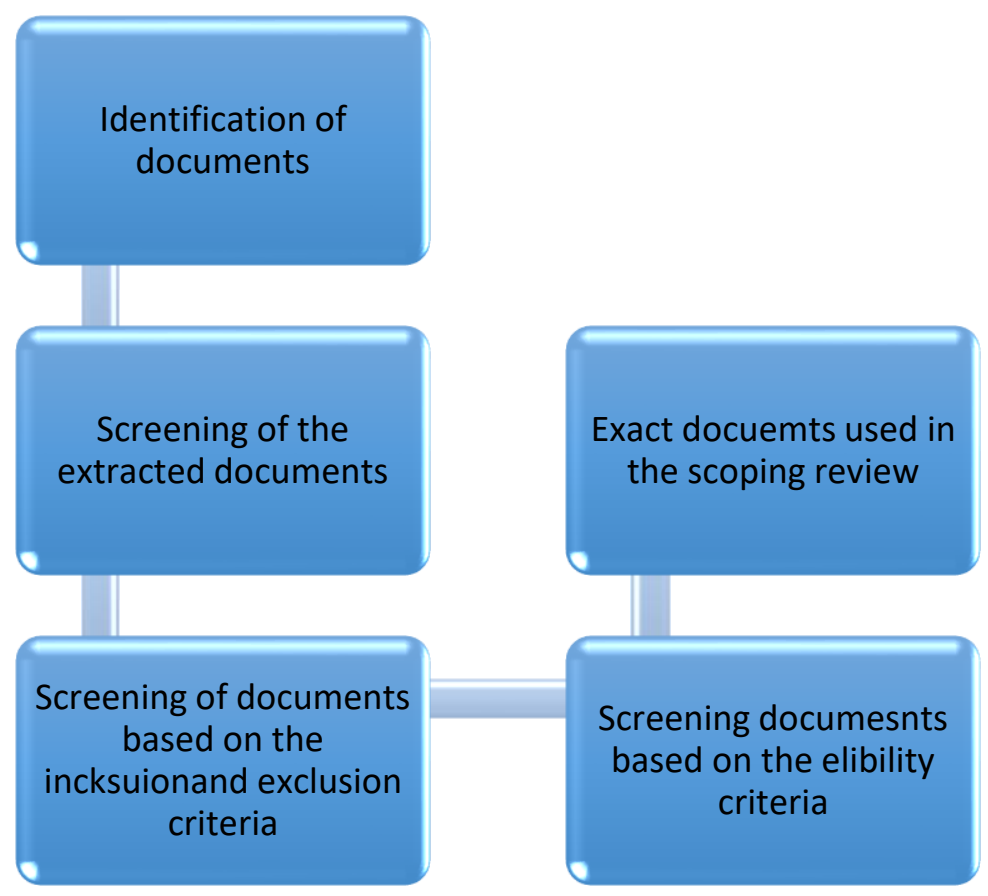

FIGURE 1. The scoping review research process

\section{Scoping Horizon}

The articles or studies used for those particular scoping reviews was those from the period between 1st, January to 30th May 2020. The time period from which the articles used in this scooping review were selected, was the most ideal since it covers a broader timeframe in which the new coronavirus diseases has immensely spread to the different parts of the world. It is argued that the studies conducted before 1st January 2020 are not so much significant about the dynamics of COVID-19 hence including them in the scoping review could affect the quality of the results.

\section{Study selection}

The articles included in this scoping review were searched from five major databases that included; Medline, Google Scholar, Web of Science (WoS), and ProQuest. These databases were selected for use in searching the different articles mainly because they harbor a great number of scholarly articles, journals, empirical studies, and reports, among other data sets or documents that can be easily accessed for usage in scoping reviews. The key search words used during the search process include; "COVID-19", "Coronavirus", "manifestations", "SARS-COV-2", "dynamics" and "prevention". The search was only done on articles written in the English language and the last search of literature was conducted on 29th May, 2020. It should also be noted that different reference lists of the identified articles from trusted websites or databases were based on to establish additional reports on COVID-19 in order to broaden the scoping review.

\section{Eligibility Criteria}

The eligibility of articles included in this particular scoping review research was based on the inclusion and exclusion criteria used in selecting the articles. The inclusion and exclusion criteria are an important element of a scoping review since it helps to establish what type of information is to be used in the review and differentiate or isolate from the less important or unwanted information. For this particular scoping review, an inclusion of all scholarly articles or empirical studies conducted about coronavirus between January and May 2020 was made without restrictions. The details of the inclusion and exclusion criteria for this particular scoping review is presented in Table 1 below; 
Table 1. Inclusion and exclusion criteria for the scoping review

\begin{tabular}{ll}
\hline Inclusion criteria & Exclusion criteria \\
\hline Papers published between January and late May, 2020 & $\begin{array}{l}\text { Papers published before January 2020 or } \\
\text { after May 2020 }\end{array}$ \\
\hline Peer reviewed or empirical articles on COVID-19 & Blogs and Wikipedia \\
\hline Papers must explain different dynamics of COVID-19 & Papers not associated with COVID-19 \\
\hline Papers based on the health perspectives of COVID-19 & Papers with no health perspectives
\end{tabular}

The inclusion and exclusion criteria were utilized ion scrutinizing the available 1,067 documents based on whether they tackle the different dynamics of COVID-19. The inclusion criteria clearly explained the attributes the articles must possess in order to be considered for the scoping review whereas the exclusion criteria clarified on the characteristics found in essential documents that disqualify them from being considered for the study as explained in Table 1. The eligibility criteria helped in the elimination of 873 documents leaving a sample of 194 documents.

\section{RESULTS}

The scoping review revealed that there is a significant increase in the number of studies conducted about COVID-19 most especially concerning the transmission, and control mechanisms of the diseases. Initially, the disease had not attracted great attention mainly because it had not yet been declared a global health emergency and pandemic respectively. This justifies the reason to why before January 2020, limited research had been conducted on the new coronavirus disease (COVID-19). Most of the studies or articles about COVID-19 or SARS-CoV-2 have been, mostly conducted by Chinese scholars or researcher. This is majorly because the disease was first announced in Wuhan China thereby attracting great attention from the different Chinese researchers. However, the studies included in this scoping review were conducted from different countries mainly China and by different organizations such as WHO as presented in figure 2 .

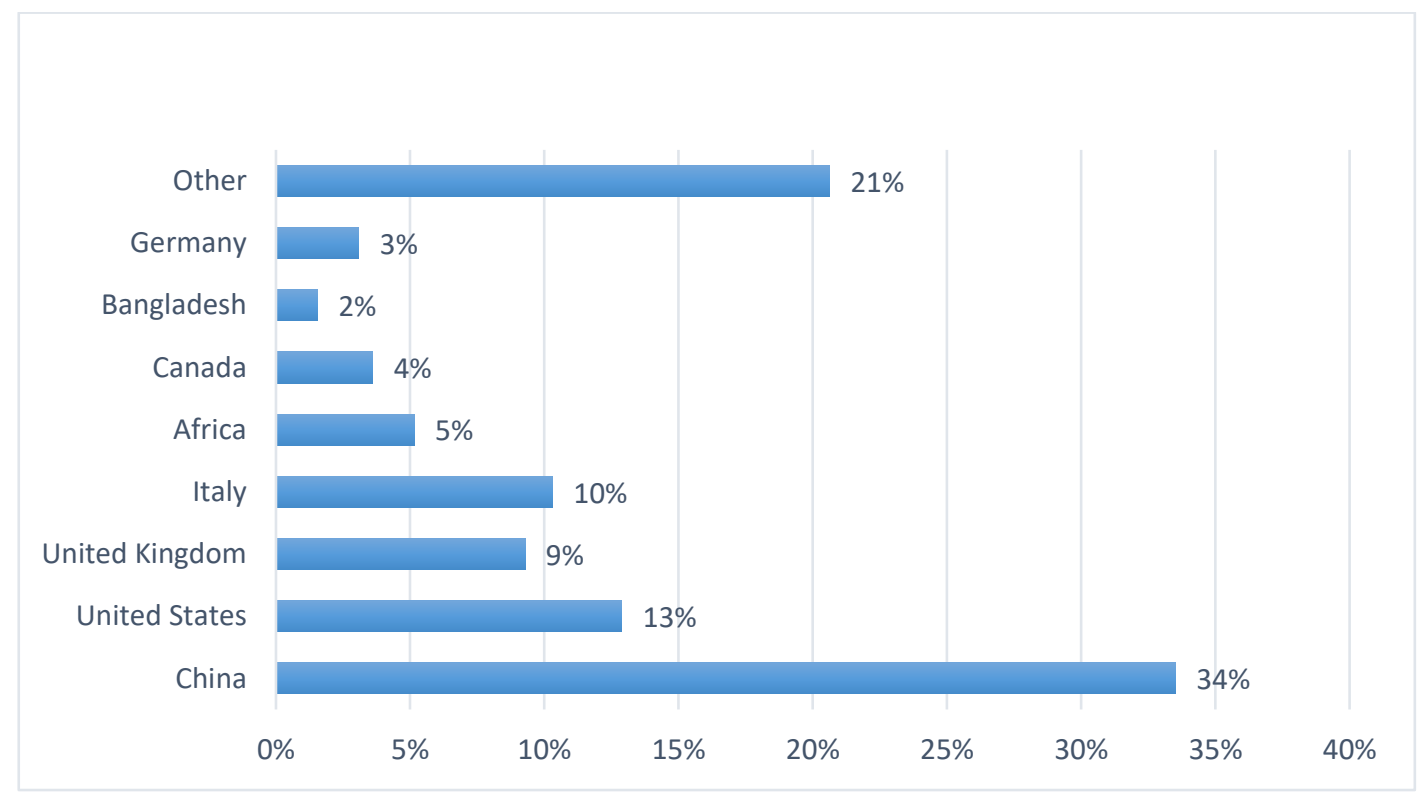

FIGURE 2. Country or origin of the articles

The new coronavirus disease (COVID-19) has for now been associated with several dynamics concerning its cause, the diverse effects it renders on human lives, and the best possible strategies that can be adopted to reduce its high global prevalence. The studies reviewed in this scoping review research involved the reporting of different dynamics of COVID-19 such as the experiences and practices associated with the 
disease, and the recommendation on how to prevent COVID-19. Other studies were associated with analyzing the different aspects of COVID-19 such as the mode of transmission, the relationship between the disease and the respiratory system, and the immediate signs and symptoms of COVID-19, among others. The key dynamics that were discovered in the different reviewed studies in this scoping review research included; the transmission of COVID-19, causes, implications, and the control and prevention strategies or mechanisms of the new coronavirus disease. Figure 3 below presents the percentage distribution of the key dynamics of features that were established per each study in relation to the new coronavirus disease.

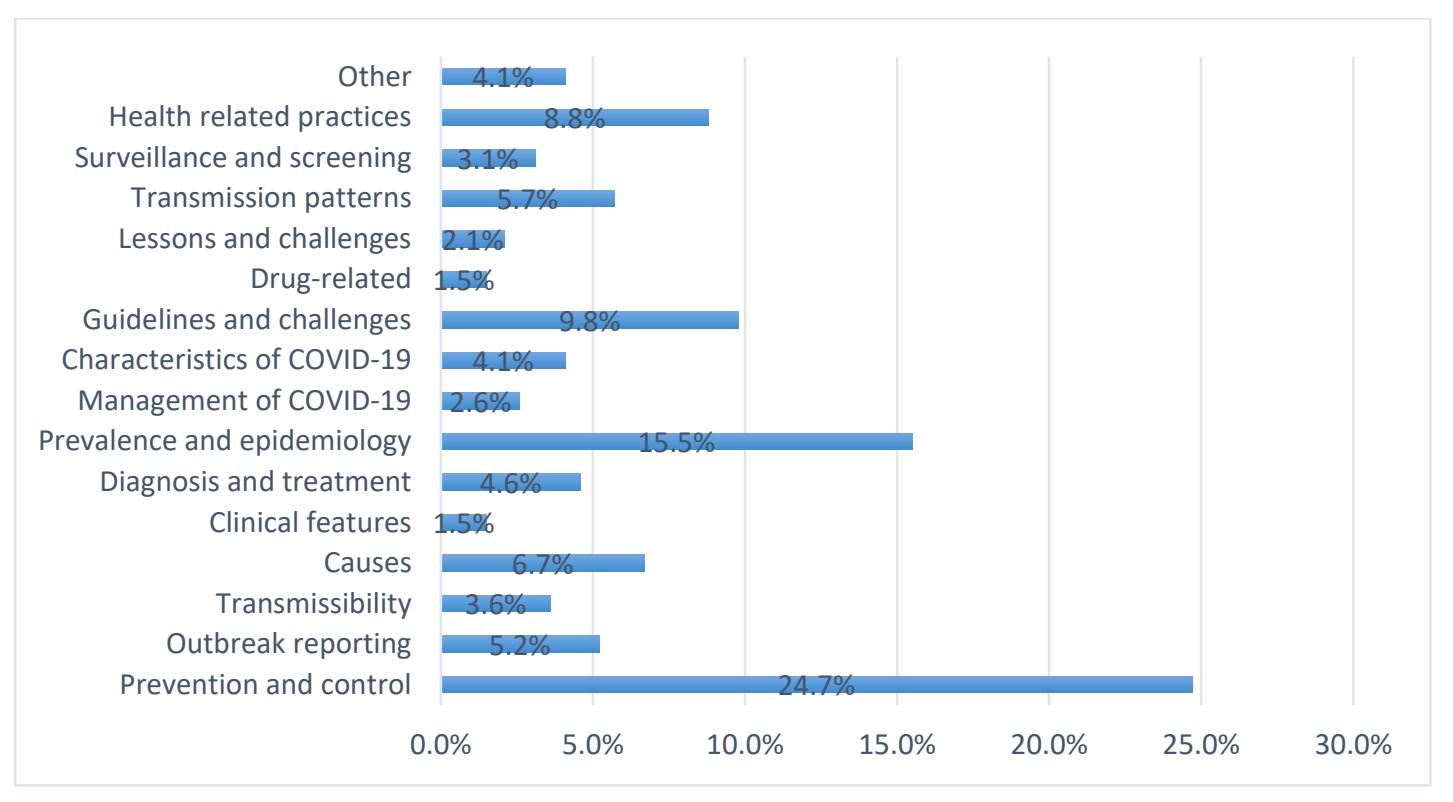

FIGURE 3. Dominant dynamics of COVID-19 in the articles included in the scoping review

The different reviewed studies were mainly publications of the Lancet $(\mathrm{n}=54)$ and these formed $27.8 \%$ of the total number of articles included in this scoping review as presented in figure 4 below. 


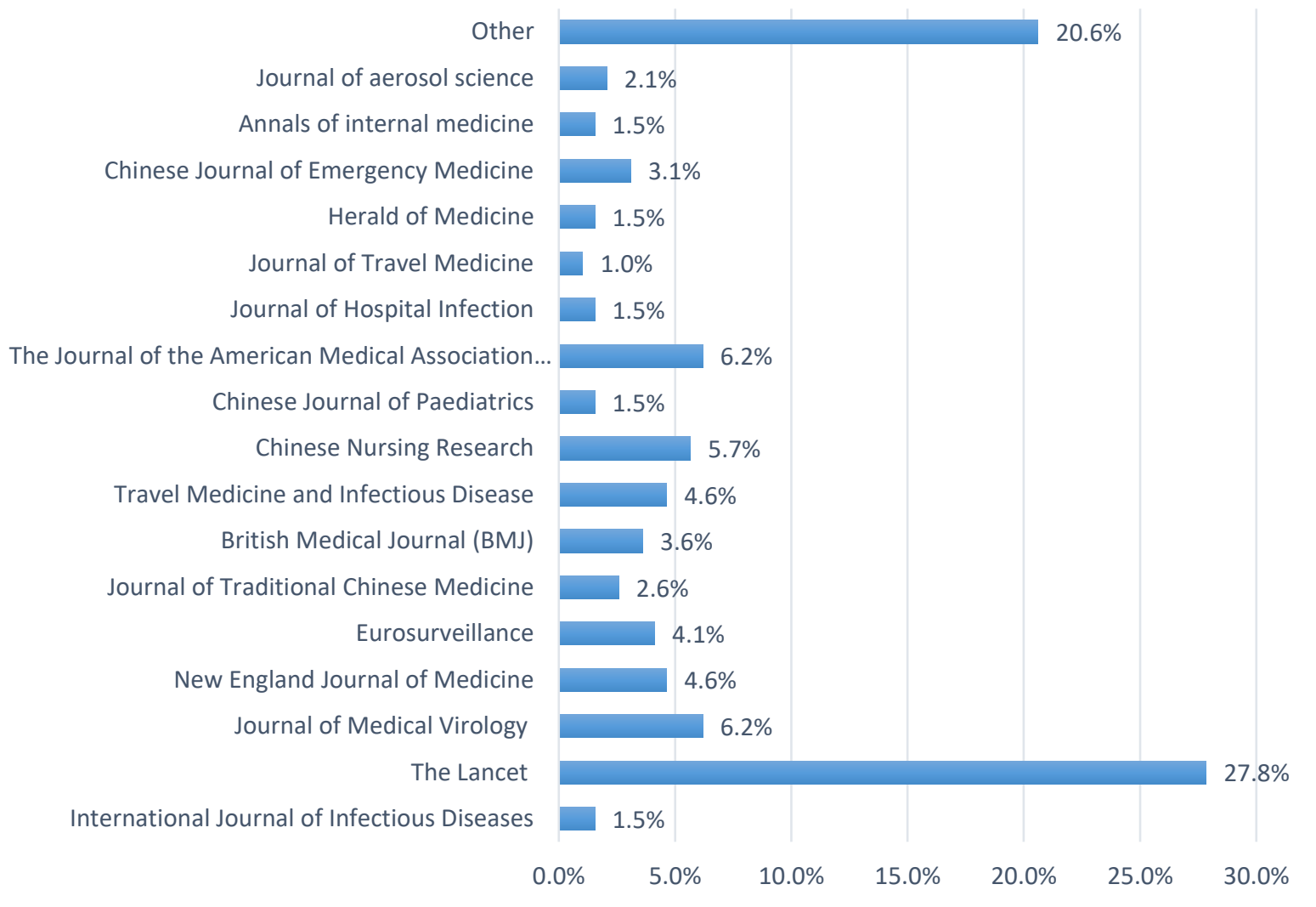

FIGURE 4. Distribution of journals or publications in the scoping review

Most of the studies reviewed ( $\mathrm{n}=76)$ were descriptive in nature representing $39.2 \%$ whereas while others were least number of studiers were analytical hence involved the use of different complicated research designs such as experimental designs, case studies, and observational designs, among others. These are always very hard to conduct which justifies the reason to why most of the studies reviewed were descriptive in nature. The importance with using descriptive designs is that they enable the research to conduct and complete the study in the shortest time possible as completed to analytical studies. The highest number of studies was extracted from ProQuest $(n=98)$ followed by Google Scholar ( $n=47)$, Medline ( $n=11$ ) and Web of Science $(n=38)$. The commonest genre on which the studies focused was the control and prevention of COVID-19 which represented about $24.7 \%$ of the total articles analyzed in the scoping review research. The different articles associated with the prevalence and epidemiological aspects of COVID-19 $(n=30)$ were the second most analyzed articles representing about $15.5 \%$ of the total number of articles analyzed. The articles about the different guidelines and challenges associated with the new coronavirus disease $(n=19)$ were ranked third representing $9.8 \%$ of the total number of articles reviewed. The other articles were about the different heath practices associated with COVID-19 ( $n=17)$ such vaccine development and health implications of the disease, among others and these represented $8.8 \%$ of the total reviewed articles. Other articles focused on outbreak reporting, genetics, transmissibility, clinical features, diagnosis and treatment, management of COVID-19, characteristics of COVID-19, drugs related to COVID19, lessons and challenges, transmission patterns, and surveillance and screening mechanisms, among others as presented in figure 5 .

Scholars such as Rajkumar (2020), Kassema (2020), and Kissler et al., (2020), among many other studies, focused on classifying the different dynamics of COVID-19 such as the causes, treatment, and prevention, among other dynamics. Most of these articles focused on the causes of COVID-19 representing the largest portion of the total number of articles reviewed. However for this scoping review, the most identified articles were those that focused on the prevention and control of COVID-19 Cheng et al. (2020), Chiu et al. (2020), Boettler et al. (2020), Bartoszko et al. (2020), and Dolbeault \& Turinici (2020). Nevertheless, the 
causes of COVID-19 also fall under the different dynamics of this disease hence tend to overlap with the prevention and control mechanisms of COVID-19 that were largely focused on in this scoping review.

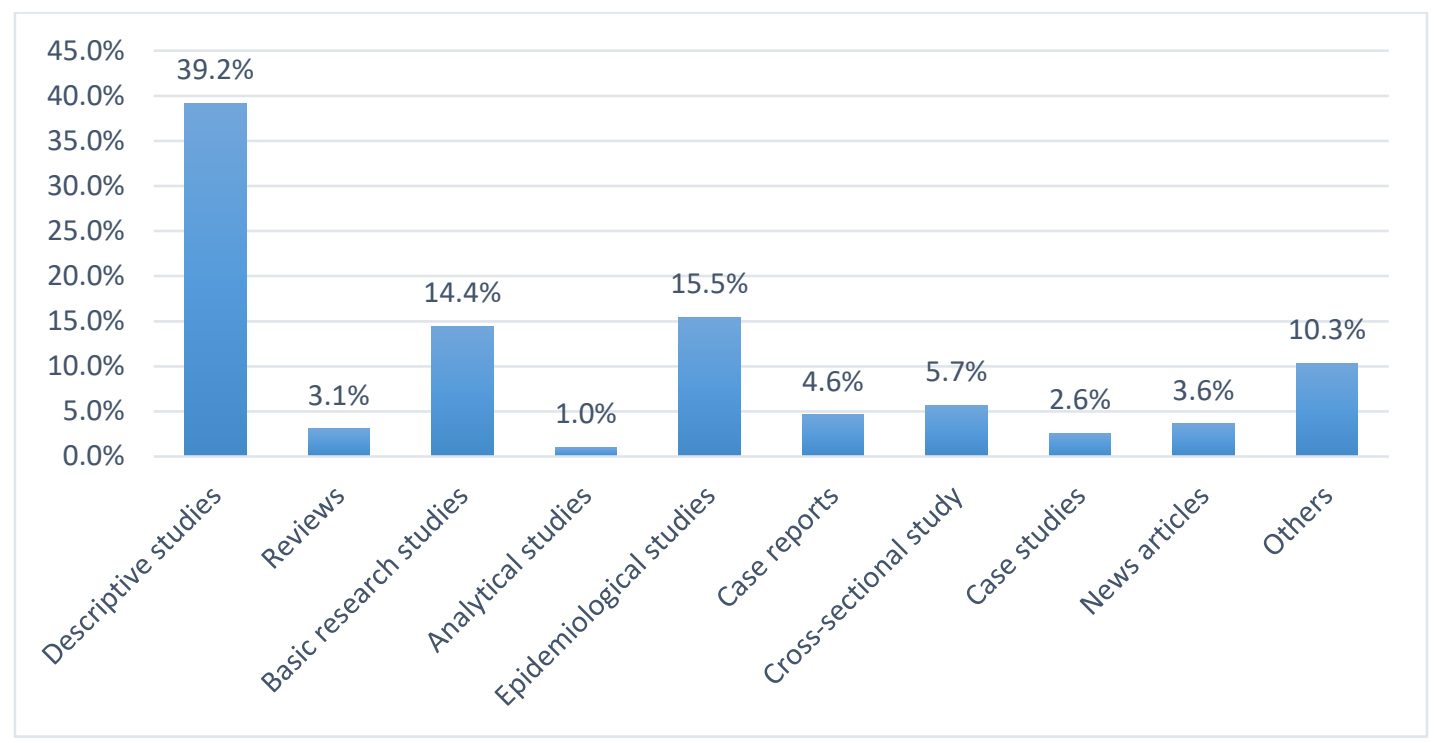

FIGURE 5. Types/characteristics of studies included in the scoping review

\section{DISCUSSION}

It The scoping review indicates that most of the studies about the dynamics of COVID-19 have been conducted in developed countries as compared to less developed countries. The results of the scoping review research show that there is an increase in the number of articles written and studies conducted about the COVID-19 since its announcement in China. Most of the studies about COVID-19 are clinical studies which is a clear indication that the disease has become a global health threat that needs more attention from different concerned stakeholders in the healthcare industry and other humanitarian agencies across the world (Verity et al., 2020). The scoping review reveals that despite that the continued increase in the number of journals or articles about coronavirus, several gaps are still existent in most of the research studies about the dynamics of COVOD-19. This therefore indicates that most academic scholars have not yet exhausted some of the important critical issues associated with COVID-19. This can be attributed to several reasons that may be related with the type of research design used, the nature of the sample size, the expertise of the researcher, and the time available to undertake most of these studies (Dolbeault \& Turinici, 2020).

The scoping review also revealed that a few studies have focused on the clinical perspective of COVID-19 which has posed a great a challenge in establishing the most effective ways of totally eradicating the disease or preventing it from spreading further to other parts of the world (Shi et al., 2020). The limited number of clinical studies about the new coronavirus disease has also affected the ability of different health practitioners to develop or establish clinical practice guidelines and health policies to use in addressing the pandemic (Spagnuolo et al., 2020). There are also gaps in the clinical studies conducted about the disease and most of these gaps are attributed to the continued spread of the disease to several countries around the world and limited empirical studies about the core dynamics of COVID-19 and the virus that causes this disease (Lv et al., 2020). It is also important to note that conducting clinical research studies requires a lot of time hence it may have been difficult for researchers to exhaust all issues associated with COVID-19 in the limited time available since its recognition as a global health problem (Ahmad, \& Rathore, 2020). This therefore justifies the existence of several research gaps in the different clinical studies considered for this particular scooping review. It should be noted that when faced with a huge health problem or global health emergency that has no previous known cause, academic scholars or researchers should endeavor to conduct very many studies to establish whether the existing health interventions, policies or guidelines are effective in addressing such health emergency (Djalante, Shaw, \& DeWit, 2020). Once it is established that available public health interventions are less effective, then more experimental studies have to be conducted in order to establish the most effective mechanism for handling the health problem. However no such studies were identified in this scoping review. This indicates that researchers still have much work 
to do concerning the need to establish additional public health or clinical interventions to address COVID19 provided the existing mechanisms fail to eradicate the disease (Asyary \& Veruswati, 2020).

The scoping review research found out that over 20 percent of the different studies articles that have been conducted in the past about the new coronavirus diseases have been availed to the general public on a global basis by posting them on free and easily accessible websites (Mallineni et al., 2020). The practice of availing to the public the different articles or studies about COVID-19 is very essential most especially in such times of health emergencies or pandemics that require every person to be updated about the different dynamics of the health problem. Most of the studies conducted in the past have revealed that preprints are so effective in addressing different health emergencies or outbreaks of several infectious diseases such as COVID-19 (Ramelli \& Wagner, 2020).

The scoping review also established that the different research topics in the reviewed studies or articles were associated with a number of similarities and differences. Most of the research as presented by the scholars was greatly focused on establishing the origin or exact cause of the new virus. On the contrary most of the epidemiological studies included in the scoping review were much concerned with the general transmissibility of the virus. Past reviews and reports about the virus also offered important information about COVID-19 and the details of its outbreak, whereas the guidelines or guidance phrases offered different recommendations on how to address the new coronavirus disease (Tai et al., 2020).

Most of the clinical statements included in the reviewed studies encompass several recommendations that can be help to optimize patient care with basis on existing evidence about the benefits and harms of alternative care options as evidenced in Feng et al. (2020), Kissler et al. (2020), Agbehadji et al. (2020) and Kong (2020). The different clinical guidelines are an important tool to healthcare workers since they act as a basis for understanding different actions to professionally address an existing health emergency. The different pieces of advice contained in the guidelines can help clinicians to understand the possible ways to undertake different tasks associated with handling patients suffering from the disease under review. For example following the outbreak of COVID-19, several organizations such as WHO and different health institutions across the world availed a great number of strategies to help in addressing the global pandemic (WHO, 2020). These guidelines have some extent helped in managing the disease since most countries such as China that were initially greatly affected by the disease have registered a high number of recoveries. However, it should be noted that most of these documents provided the organizations did not fulfil most of the important principles of evidence-based practice of the healthcare system.

A comprehensive review of the articles or studies included in the scoping review revealed that most of the guidelines presented by researchers failed to grade the quality of evidence and strength of recommendations and this is evidenced in studies of Edwards et al. (2020), Dantas et al. (2020), and Konda et al. (2020) as well as Hall, Scott \& Gössling, (2020). In this case, the guidelines owed to the emerging health emergency but it is always advisable for such guidelines or prevention mechanisms to be considered along with the different limitations. Different health based international organizations such as WHO have continuously focused on establishing different guidelines used in offering advice in case of any health emergencies. The reports produced by WHO (2020) suggest that when any global health problem arises, a rapid review of the problem should be immediately conducted in order to establish the different dynamics associated that particular problem. It is important to note that establishing the different dynamics of a global health emergency or pandemic helps to devise mechanisms or strategies that can be based on to address the existing health problem. However, conducting reviews on the dynamics or features of a global health emergency is always a challenge. This is mainly due to limited time available to establish the most competent personnel required to participate in the development of different guidelines for the review.

\section{CONCLUSIONS and RECOMMENDATIONS}

The scoping review conducted on the dynamics of the new coronavirus disease (COVID-19) shows that a number of research studies and review articles have been written since its announcement as a global emergency and pandemic in respectively. The number of articles about Ccovid-19 have gradually increased though most of the studies have been conducted in China since it is the country that was affected first and devised the initial; mechanisms or strategies of curbing the deadly coronavirus diseases. Other countries have also embraced research in COVID-19 most especially concerning the ways through which the disease is spread and how best it can be controlled. It is however evident from the articles reviewed that most of them, most especially the clinical based studies, lack diversity concerning the spreads and the immediate or most effective control and prevention strategies of COVID-19. Nevertheless, this scoping review helps to identify several gaps that are existing in most of the past studies conducted about COVOD-19 which helps researchers to establish ways on how to fill such gaps. 
It From the articles reviewed about the dynamics of COBVID-19, there is a clear need for more research especially about the most effective mechanisms for stopping further spread of COVID-19. Most of the studies conducted in the past about the disease have only focused on the ways through which the disease has spread to various parts of the world and the possible ways of reducing it, but lack enough evidence on how it will be stopped in the near future. This therefore calls for more clinical studies to establish the most ideal vaccine that can be used to eradicate the disease and consequently improve on the immunity of the global community since it is the only way of strengthening individual health against any possible contraction of the new coronavirus disease. It is also important that academic scholars or researchers continue to review more emerging studies or articles being conducted or written about COVID19. This will help to establish and understand more evidence about the disease and consequently help to solve the problem of overlapping work when conducting new research studies. Furthermore, more clinical studies or scoping reviews will help to improve evidence that is very essential for continued development of different guidelines and policies for clinical research and wellbeing of global healthcare system. In this case evidence presented through different studies helps different stakeholders in the healthcare industry to understand the dynamics of emerging pandemics and consequently devise different ways on how to address such pandemics. Due to the high variability in the conduct of scoping reviews, there is need for proper methodological standardization which will help to improve the utility and general strength of the evidence.

\section{REFERENCES}

Agbehadji, I. E., Awuzie, B. O., Ngowi, A. B., \& Millham, R. C. Review of Big Data, Artificial Intelligence and Nature-Inspired Computing Models for Performance Improvement towards Detection of COVID19 Pandemic Case and Contact Tracing. https://www.researchgate.net/profile/Israel_Agbehadji2/publication/341079054_Review_of_Bi g_Data_Artificial_Intelligence pdf

Ahmad, I., \& Rathore, F. A. (2020). Neurological manifestations and complications of COVID-19: A Literature Review. Journal of Clinical https://www.sciencedirect.com/science/article/pii/S096758682031078X

Arksey, H., \& O'Malley, L. (2005). Scoping studies: towards a methodological framework. International $\begin{array}{lllll}\text { journal of } & \text { social } & \text { 19-32. }\end{array}$ https://www.tandfonline.com/doi/abs/10.1080/1364557032000119616

Asyary, A., \& Veruswati, M. (2020). Sunlight exposure increased Covid-19 recovery rates: A study in the central pandemic area of Indonesia. Science of The Total Environment, 139016. https://www.sciencedirect.com/science/article/pii/S004896972032533X

Bartoszko, J. J., Farooqi, M. A. M., Alhazzani, W., \& Loeb, M. (2020). Medical masks vs N95 respirators for preventing COVID-19 in healthcare workers: A systematic review and meta-analysis of randomized trials. Influenza and other viruses. https://onlinelibrary.wiley.com/doi/abs/10.1111/irv.12745

Bhuiyan, A. I., Sakib, N., Pakpour, A. H., Griffiths, M. D., \& Mamun, M. A. (2020). COVID-19-related suicides in Bangladesh due to lockdown and economic factors: case study evidence from media reports. International Journal of Mental Health and Addiction. https://link.springer.com/content/pdf/10.1007/s11469-020-00307-y.pdf

Boettler, T., Newsome, P. N., Mondelli, M. U., Maticic, M., Cordero, E., Cornberg, M., \& Berg, T. (2020). Care of patients with liver disease during the COVID-19 pandemic: EASL-ESCMID position paper. JHEP Reports, 100113. https://www.sciencedirect.com/science/article/pii/S2589555920300471

Cheng, Y., Luo, R., Wang, K., Zhang, M., Wang, Z., Dong, L., ... \& Xu, G. (2020). Kidney disease is associated with in-hospital death of patients with COVID-19. Kidney international. https://www.sciencedirect.com/science/article/pii/S0085253820302556

Chiu, P. W. Y., Ng, S. C., Inoue, H., Reddy, D. N., Hu, E. L., Cho, J. Y., ... \& Wang, H. P. (2020). Practice of endoscopy during COVID-19 pandemic: position statements of the Asian Pacific Society for Digestive Endoscopy (APSDE-COVID statements). Gut, 69(6), 991-996. https://jamanetwork.com/journals/jamaotolaryngology/article-abstract/2764032

Cowling, B. J., Ali, S. T., Ng, T. W., Tsang, T. K., Li, J. C., Fong, M. W., ... \& Wu, J. T. (2020). Impact assessment of non-pharmaceutical interventions against coronavirus disease 2019 and influenza in Hong

Dantas, G., Siciliano, B., França, B. B., da Silva, C. M., \& Arbilla, G. (2020). The impact of COVID-19 partial lockdown on the air quality of the city of Rio de Janeiro, Brazil. Science of The Total Environment, 729, 139085. https://pubmed.ncbi.nlm.nih.gov/32335408/ 
Djalante, R., Shaw, R., \& DeWit, A. (2020). Building resilience against biological hazards and pandemics: COVID-19 and its implications for the Sendai Framework. Progress in Disaster Science, 100080. https://www.mdpi.com/1660-4601/17/7/2309/pdf

Dolbeault, J., \& Turinici, G. (2020). Heterogeneous social interactions and the COVID-19 lockdown outcome in a multi-group SEIR model. arXiv preprint arXiv:2005.00049.https://hal-pasteur.archivesouvertes.fr/pasteur-02548181/document

Edwards, S. P., Kasten, S., Nelson, C., Elner, V., \& McKean, E. (2020). Maxillofacial trauma management during COVID-19: multidisciplinary recommendations. Facial Plastic Surgery\& Aesthetic Medicine. https://pmj.bmj.com/content/early/2020/04/03/postgradmedj-2020-

137781?utm_source=TrendMD\&utm_medium=cpc\&utm_campaign=BMJ\%27s_Coronavirus_(covi d-19)_Hub_TrendMD_0

Feng, Y., Marchal, T., Sperry, T., \& Yi, H. (2020). Influence of wind and relative humidity on the social distancing effectiveness to prevent COVID-19 airborne transmission: A numerical study. Journal of aerosol science,

105585. https://www.sciencedirect.com/science/article/pii/S0021850220300744

Givi, B., Schiff, B. A., Chinn, S. B., Clayburgh, D., Iyer, N. G., Jalisi, S., ... \& Parker, N. (2020). Safety recommendations for evaluation and surgery of the head and neck during the COVID-19 pandemic. JAMA Otolaryngology-Head \& Neck Surgery. https://jamanetwork.com/journals/jamaotolaryngology/article-abstract/2764032

Hall, C. M., Scott, D., \& Gössling, S. (2020). Pandemics, transformations and tourism: be careful what you $\begin{array}{llll}\text { wish for. Tourism 1-22. } & \end{array}$ https://www.tandfonline.com/doi/abs/10.1080/14616688.2020.1759131

Holmes, E. A., O'Connor, R. C., Perry, V. H., Tracey, I., Wessely, S., Arseneault, L., ... \& Ford, T. (2020). Multidisciplinary research priorities for the COVID-19 pandemic: a call for action for mental health science. The Lancet Psychiatry. https://www.sciencedirect.com/scienc 